\title{
Effect of Magnetic Treatment on Water Permeability Through a Semi-Permeable Membrane
}

\author{
Vladimir Zlotopolski \\ United Research Labs, San Marcos, USA
}

\section{Email address: \\ vzlotopolski@att.net}

\section{To cite this article:}

Vladimir Zlotopolski. Effect of Magnetic Treatment on Water Permeability Through a Semi-Permeable Membrane. American Journal of Water Science and Engineering. Vol. 3, No. 2, 2017, pp. 28-33. doi: 10.11648/j.ajwse.20170302.12

Received: May 16, 2017; Accepted: June 20, 2017; Published: July 21, 2017

\begin{abstract}
Magnetic water treatment devices (MWT), while attractive because of their safety, simplicity, environmental friendliness and effectiveness in agriculture have been difficult to assess scientifically because a single, generally accepted, repeatable and measurable indicator of their decree of impact on the physical properties of water, has not been discovered. Experimental results have shown that MWT offers many agricultural benefits and that magnetically-treated water can more easily penetrate various media such as membranes, which are generally considered excellent proxies for plant cell walls. This study evaluated how MWT changes permeability through a semi-permeable membrane, how that change is impacted by flow velocity and proposed membrane permeability as a reliable indicator of MWT effectiveness. Results obtained from this study indicated that MWT changed permeability through a semi-permeable membrane and these changes depended on water flow velocity. Results further indicated that the permeability differential in the MWT treatment group decreased by almost $9 \%$ at low-flow velocities (laminar regime; $\mathrm{Re}<1000$ ) to $2.3 \%$ at the high-flow velocities, compared to control (turbulent regime; $\mathrm{Re}>4000$ ). At low-flow velocities, the electro-conductivity of MWT and the control group were statistically different at $\mathrm{p} \leq$ 0.01 . However, at higher-flow velocities, the difference between MWT and the control group was smaller and a statistically sufficient level was reached only at $p \leq 0.05$ and $p \leq 0.10$. The differences observed between the low, and high-flow velocity treatment groups was somewhat expected as high flow rates reduce the retention time of water in the treatment area and thus reduces the efficiency of magnetic treatment. These results also provide a clear indication that water has been impacted by MWT and demonstrate the degree that water has been impacted by MWT under various flow rates.
\end{abstract}

Keywords: Permeability, Magnetic Treatment, Water, Flow Velocity

\section{Introduction}

Devices which alter the physical properties of water using either permanent, or electro-magnets attract special attention due to their safety, simplicity and environmental friendliness [1]. MWT is currently used in many countries to improve water productivity, increase crop yield, induce seed germination and benefit the health of livestock.

MWT changes the molecular structure of water. According to Chang and Weng [2] the number of hydrogen bonds in water molecule clusters (or hydrates) increases by $0.34 \%$ after MWT. In the same study, it was shown that water surface tension was decreased and viscosity increased with the application of MWT. Cho and Lee [3] investigated the effect of MWT on surface tension and flow-visualization of dye behavior in hard water samples. They found that water hardness did not significantly affect the reduction of the surface tension of MWT. At the same time, the surface tension effect of MWT decreased as the number of colloidal particles increased. In their dye-injection experiment they noted that dye drops in the untreated water rapidly spread out along the radial direction; in other words, dye did not penetrate the untreated water but stayed on the surface of the water indefinitely. But when water passed through magnetic treatment, the dye drops quickly fell through the water. Controversial results were obtained by Amiri and Dadkhah [4] who also observed a reduction of surface tension due to MWT. However, after more than 200 tests over a six-month period, they concluded that the surface tension of water was too sensitive to experimental conditions to be considered a safe and reliable indicator for studying the effect of magnetic fields on water. Gang et. al. [5] investigated different water volumes $(25,50,100 \mathrm{cc})$ which were exposed to different 
magnetic fields for one hour. The diffusion velocity of India ink in $80 \mu \mathrm{L}$ in water was then measured every 15 minutes. A sudden increase by a factor of two in diffusion velocity as a function of exposed volume was observed for water treated by a static 1,600 $\mathrm{G}$ magnetic field. Other authors have reported that magnetic fields have effects on different physical parameters of water such as light absorption, and zeta potential $[6,7]$.

Several attempts were made to find a numerical parameter to quantify the impact of magnetic fields on water. Bogatin [1] suggested measuring $\mathrm{pH}$ as the simplest and quickest method of magnetic treatment indication by adding $\mathrm{Ca}(\mathrm{OH})_{2}$ to the outlet water of a magnetic treatment device. The $\mathrm{pH}(\mathrm{t})$ change would theoretically reflect the process of calcium carbonate crystallization due to the device's impact on the gas content, and the shift of the carbonic acid balance, of the solution. The main disadvantage of this approach is the amount of alkali added would have to be experimentally adjusted for each type of solution. To evaluate the effect of magnetic fields on water, Otsuka and Ozeki [8] chose to measure the contact angle of water on a Pt plate. It was found that magnetic treatment decreased the contact angle from $65^{\circ}$ to $57.5^{\circ}$ only after $\mathrm{O}_{2}$ was dissolved in the water. It is noteworthy to point out that MWT alone and the addition of electrolytes $(10 \mathrm{mM})$ such as $\mathrm{NaCl}, \mathrm{KCl}$, and $\mathrm{CaCl}_{2}$ to distilled water caused no decreased contact angle effect.

Optical methods were also suggested to evaluate the degree of "magnetization" of water. Absorption spectrum of water was measured by Majeed and Salman [9]. They found that the absorption spectrum (340-1,000 nm) of distilled water was increased after exposure to a north-pole magnetic field and was decreased after exposure to a south-pole field. South-pole magnetic force appeared to make water molecules bind to each other more weakly than normal, thus giving it lower density than normal. The lower density water is more fluid and has greater biological activity. An increase in the refractive index by $0.1 \%$ under magnetic fields up to $10 \mathrm{~T}$ was found by Hosoda et al. [10]. The possible explanation for this was that the lifetime of the hydrogen bond was prolonged due to the electron delocalization of a water dimer under the magnetic field. Another method of identifying the magnetic effect on water was suggested by Kronenberg [11]. This method included a microscopic crystal count of the relative number of calcite to aragonite crystals without and with MWT.

An analysis of data from different sources shows that the efficiency of magnetic treatment depends on the strength of the magnetic field, flow rates and probably, the type of water being treated (distilled, tap, well or sea water). There are many experimental results that show that MWT is a technique that achieves high water use efficiency $[12,13]$ or, in other words, soil holds more moisture if it was irrigated by water after magnetic treatment. There are data that indicate that MWT offers many other benefits in agriculture such as increased yield, early maturity and increased fertilizer uptake [14-19]. These results could be related to various physical parameters affected by MWT such as surface tension, viscosity and water permeability through the plant cell wall. Results of experiments with diffusion velocity and dyeinjection also lead to the same conclusion. According to these data, water after magnetic treatment can more easily penetrate various media similar to membranes, which are generally considered excellent proxies for plant cell walls. Semi-permeable membranes (for example, membranes for dialysis) are very thin layers of material that allow small molecules, like oxygen, water, carbon dioxide, ammonia, glucose, amino-acids, etc., to pass through, a process known as diffusion. However, they do not allow larger molecules, like sucrose, protein, etc., to penetrate them. Osmosis is the result of diffusion across a semi-permeable membrane. These membranes are practically identical to cell walls. If two solutions of different concentration are separated by a semipermeable membrane, then the solutions will tend to diffuse across the membrane from the less concentrated to the more concentrated solution. This process is widely used by plants and it is partially responsible for the absorption of soil water and for the elevation of the liquid to the leaves of plants.

It is generally understood that magnetic fields loosen and distract the hydrate layers and films that form around potential crystalline "seed" centers in moving liquid, thus facilitating coagulation and coalescence and improving the potential for calcium carbonate crystal formation in solution rather than on surfaces in hard water [1]. In this case, flow velocity plays a very important role. Experiments conducted with hard water have shown that increased water flow, with resultant turbulence and whirlpool effects, favor the formation of aragonite over calcite crystal formation [20]. Kronenberg [11] believes that permanent magnets do not have enough energy to promote formation of calcium carbonate in water and additional required energy can only be delivered by the kinetic energy of the flowing water. For this reason, no effect can be expected if the water does not move. Calculations conducted by Kobe et al. [21] supported this theory. His calculations suggested that the energy $(28 \mathrm{eV})$, which is required to bridge the gap between the ground electronic states of the calcite and the aragonite forms, can only be provided by a magnetic field of $45 \mathrm{~T}$. However, the presence of strong electric and magnetic fields is inherent in the motion of the fluid and the fluid can exchange energy with the electromagnetic field. As a result, the required magnetic field of $45 \mathrm{~T}$ is easily met by moving liquid in the presence of a typical magnetic field of 1-2 $\mathrm{T}$.

A different conclusion regarding the influence of flow was reached by Gang et al. [5] where spring water was treated by a Raythenon horseshoe magnet with the strength of 1,600 Gauss for one hour in the complete absence of water flow. In that study, an increase by a factor of two in diffusion velocity as a function of the exposed volume was noted. In this case, unlike experiments with hard water and crystal deposition, flow rate did not have any impact on diffusion velocity.

So, it was quite logical to evaluate how magnetic treatment changes water permeability through a semi-permeable membrane and use these changes to provide a clear indication that water has been impacted by MWT and 
evaluate the degree that water has been impacted by MWT. Also, considering the fact that flow rate has been shown to influence certain proposed MWT mechanisms of action, it was appropriate to additionally correlate flow rates to the observed changes in water permeability through a semipermeable membrane. These are the purposes of this experiment.

\section{Materials and Methods}

Portions of semi-permeable cellulose tubing (16 $\mathrm{mm}$ diameter and $25 \mathrm{~mm}$ flat; pore size 14,000 Da MWCO) from Science First was used to measure permeability through a semi-permeable membrane. An EC-meter (sensION+EC7, $\mathrm{HACH}$ ) equipped with a magnetic stirrer was used to measure the electro conductivity of solutions. The EC-meter was checked and calibrated (if needed) before each experiment according to the recommended procedure. A small piece of dialysis tubing $(10 \mathrm{~cm}$ long $)$ was cut and filled out by $0.1 \mathrm{~N} \mathrm{NaCl}$ solution $(7.0 \mathrm{ml})$. This tubing was placed in a glass beaker with $500 \mathrm{ml}$ of tap water for $30 \mathrm{~min}$. The top of the tubing was open to the air. After 30 minutes, the tubing was removed from the beaker and the solution was transferred by a plastic $10 \mathrm{ml}$ syringe into a $10 \mathrm{ml}$ glass vial to measure the final EC. The vial was placed on the magnetic stirrer of the EC-meter and the electro conductivity of the solution was determined. These experiments were conducted with tap water (Table 1) at a temperature of $23 \pm 1 \mathrm{C}$. Before the measurement, the tap water was passed through a magnetic field at different flow rates. Each experiment was repeated at least six times. The experiment with the control group (no magnetic treatment) was repeated 15 times.

Table 1. Water Parameters.

\begin{tabular}{lllllllll}
\hline $\mathbf{C a}, \mathbf{p p m}$ & $\mathbf{M g}, \mathbf{p p m}$ & $\mathbf{K}, \mathbf{p p m}$ & $\mathbf{N a}, \mathbf{p p m}$ & $\mathbf{C l}, \mathbf{p p m}$ & $\mathbf{S O 4}, \mathbf{p p m}$ & HCO3, $\mathbf{p p m}$ & $\mathbf{p H}, \mathbf{u n i t s}$ & $\mathbf{E C}, \mathbf{m S} / \mathbf{c m}$ \\
\hline 76.8 & 25.1 & 5.2 & 105.8 & 97.6 & 226.1 & 54 & 7.9 & 0.9 \\
\hline
\end{tabular}

The MWT was applied using the Wellpure Water Treatment System ${ }^{\mathrm{TM}}$ physical water treatment device. This system treats water by utilizing a number of modalities, including a magnetic modality (Figure 1). Normally the magnetic component of the system contains 18 ring-shaped, permanent, rare-earth, metal magnets placed in two polycarbonate flanges oriented with their respective polarities in opposition to each other.

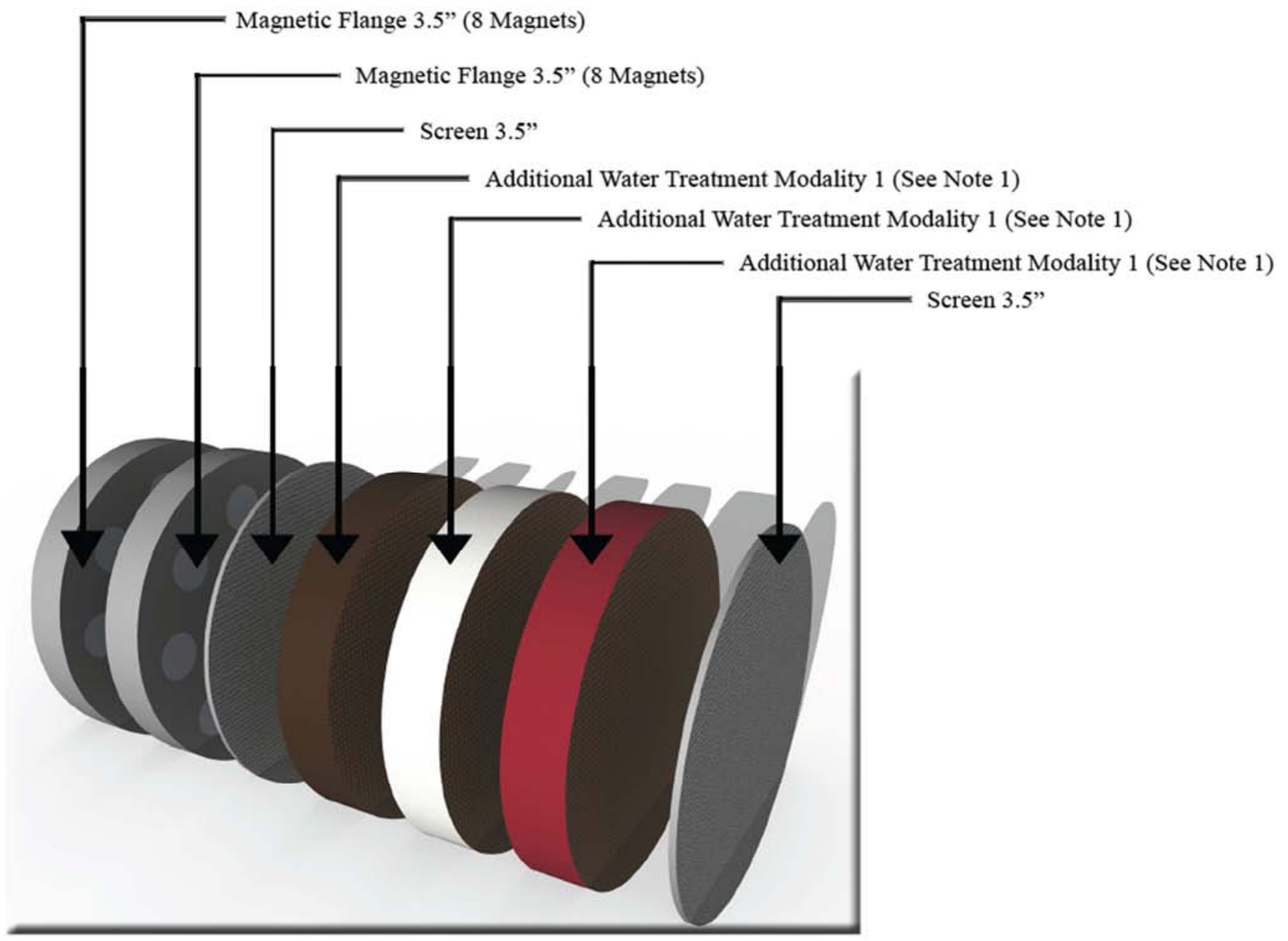

(Note 1 - These additional treatment modalities are not researched in this study)

Figure 1. The cross section of the Wellpure Water Treatment System ${ }^{T M}$. 
The distance between the two flanges is $4 \mathrm{~mm}$ and each magnet has a $12 \mathrm{~mm}$ inner hole. The design forces all water moving through the system to pass through the magnets' inner holes.

Only the magnetic modality of the device was investigated in this study. A modified magnetic device was used with 2 polycarbonate flanges with 18 ring-shaped permanent magnets. The magnetic field strength was measured by a Gaussmeter Model GM-2 (AlphaLab Inc.) and it ranged from 3,600 $\mathrm{G}$ (close to the edges) to $700 \mathrm{G}$ (in the middle of the hole) for each magnet. Two experimental apparatus were assembled to pass water at different flow rates through the modified device. The first apparatus passed water from a 100L tank through the modified device at flow rates up to 5 gpm. A 1L cylinder and stopwatch timer was used to measure flow rates. A second apparatus was assembled for larger flow rates up to $20 \mathrm{gpm}$. In this case, a 2,000L tank and a flow meter with a measurement range from 0-25 gpm (King Instruments) were used. Water was passed only one time through the modified magnetic device. Samples of treated water were collected and water permeability through the semi-permeable membrane was immediately measured. A one-way analysis of variance (ANOVA) was used to determine the significant differences between treated and not treated groups. Standard error bars were included.

\section{Results and Discussion}

Results from this study indicated that flow rates and magnetic treatment had a direct impact on water penetration through the semi-permeable membrane. Table 2 presents the observed experimental data and computed statistical data, velocity and Reynolds numbers.

Table 2. Experimental results and variance analysis of water permeability.

\begin{tabular}{llllllllll}
\hline Control/ Test \# & Flow, gpm & Number of repeats & Mean EC, mS/cm & STD & FOV & F CV & $\mathbf{p} \leq$ & V, cm/sec & Re \\
\hline 1 & 2 & 3 & 4 & 5 & 6 & 7 & 8 & 9 & 10 \\
Control 1 & $1.03-3.67$ & 15 & $3.59 \pm 0.04$ & 0.16 & - & - & - & - & - \\
1 & 3.67 & 9 & $3.42 \pm 0.02$ & 0.06 & 9.237 & 7.95 & 0.01 & 22.7 & 2702 \\
2 & 2.92 & 6 & $3.36 \pm 0.04$ & 0.09 & 10.799 & 8.19 & 0.01 & 18.1 & 2151 \\
3 & 1.31 & 12 & $3.46 \pm 0.04$ & 0.14 & 4.807 & 4.24 & 0.05 & 8.1 & 964 \\
4 & 1.03 & 6 & $3.30 \pm 0.02$ & 0.03 & 18.871 & 8.19 & 0.01 & 6.4 & 758 \\
Control 2 & $10-20$ & 15 & $3.80 \pm 0.03$ & 0.12 & - & - & - & - & - \\
5 & 20 & 9 & $3.71 \pm 0.04$ & 0.12 & 3.164 & 2.945 & 0.10 & 123.8 \\
6 & 10 & 6 & $3.64 \pm 0.05$ & 0.12 & 6.637 & 4.38 & 0.05 & 61.9 \\
\hline
\end{tabular}

$\left(\mathrm{F}^{\mathrm{OV}}, \mathrm{F}^{\mathrm{CV}}\right.$ - observed and critical values from F distribution; $\mathrm{p}$ - level of significance; STD - standard deviation; Re - Reynolds number $)$

Experiments with control group 1, the low flow rate control group, were conducted at flow rates ranging from 3.78 - 13.87 L/min (1.03-3.67 gpm). The mean EC number observed for this group was $3.59 \pm 0.04 \mathrm{mS} / \mathrm{cm}$. Control group 2 , at higher flow rates of between 37.8-75.6 L/min (10-20 gpm) had a mean EC number of $3.80 \pm 0.03 \mathrm{mS} / \mathrm{cm}$. The higher EC value of control group 2 at the higher range of flow rates can be explained by higher levels of water turbulence and possible dissolving of air which interferes with water penetration through the membrane. Data obtained after magnetic treatment at different flow rates are presented as Test \# 1-4 for low flow rates and Tests \# 5-6 for high flow rates. These data show that magnetic treatment changed water permeability through the semi-permeable membrane and, as a result, reduced the mean EC.

The most significant effect was reached at the low flow rate. Columns number 6 and 7 present critical and observed values from $F$ distribution at different level of significance $p$ (column \#8).

At low flow rates, the electro-conductivity of MWT and the control group were statistically different at $\mathrm{p} \leq 0.01$.
However, at higher flow rates, the difference between MWT and the control group was smaller and a statistically sufficient level was reached only at $p \leq 0.05$ and even $p \leq$ 0.10 . This result was somewhat expected as high flow rates reduce the retention time of water in the treatment area and thus reduces the efficiency of magnetic treatment.

The relationship between permeability differential and flow velocity was presented as Reynolds number (Figure 2). The permeability differential was calculated as the EC difference between the control and MWT groups divided on average between these groups:

$$
P D=100 *\left(\sum_{i=1}^{n} \frac{E C c}{n}-\sum_{j=1}^{m} \frac{E C m t w}{m}\right) / \frac{1}{2}\left(\sum_{i=1}^{n} \frac{E C c}{n}+\sum_{j=1}^{m} \frac{E C m t w}{m}\right)
$$

Where ECc - electroconductivity of control group; ECmtw -electroconductivity of MWT group; $\mathrm{n}-$ number of measurements in control group (15); $\mathrm{m}$ - number of measurements in MWT group. 


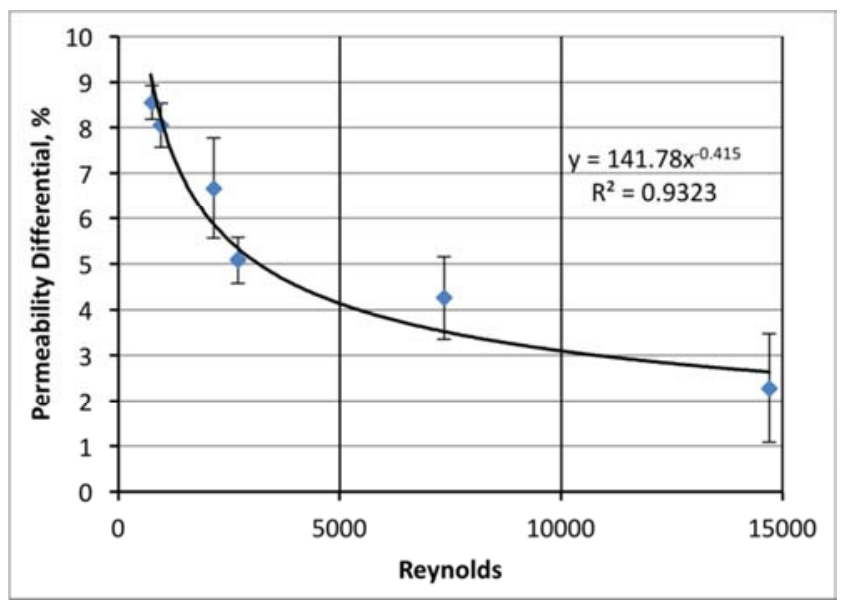

Figure 2. Relationship between Permeability Differential and Reynolds number.

The permeability differential in the treatment group decreased by almost $9 \%$ at the low-flow velocity (laminar regime; $\operatorname{Re}<1000)$ to $2.3 \%$ at the high-flow velocity, compared to control (turbulent regime; Re $>4000$ ). These results reflect an opposite outcome to that suggested by the theory of calcite/aragonite formation described above where velocity increased the rate of aragonite formation by the addition of the kinetic energy of higher velocity water flow and may indicate that the mechanism of permeability increase through a semi-permeable membrane is different than that which is apparently associated with the relative number and type of crystals formed in hard water under MWT. A more likely explanation for the membrane permeability effect is related to the mechanisms that favor lower, or no water flow or that are not impacted by water flow variables at all, but rather favor variables such as volume of exposed water, time of exposure and volume and type of dissolved gases. As previously cited, these mechanisms include surface tension decreases and diffusion velocity increases.

Another explanation may be related to a reduction of dissolved gases in water after MWT. Bondarenko and Gak [22] observed a $25-30 \%$ enhancement of degassing after magnetic treatment. Undoubtedly, in addition, removing micro bubbles from water will promote improved penetration of liquid through a semi-permeable membrane. Moreover, these two mechanisms could be related. Otsuka and Ozeki [8] investigated the change in contact angle of distilled water on a platinum plate. It was shown that the contact angle of vacuum-distilled water was unchanged by magnetic treatment. However, when MWT was performed after $\mathrm{O}_{2}$ (or air) was dissolved in the water, the contact angle decreased dramatically. Further, when their vacuum-distilled, $\mathrm{O}_{2}$ dissolved and MWT was subjected to Raman spectrometry they noted that oxygen exposure prior to MWT had no effect on the Raman spectrum. However, after MWT all Raman bands were markedly strengthened and a significant "shoulder" in the range of 480-540 nm appeared. This shoulder was ascribed to oxygen clathrate-like hydrates. This suggests that MWT, in the presence of dissolved oxygen, produces a fundamental change in water's hydrate clusters' chemical states.

\section{Conclusion}

Experimental results indicated that the permeability of water through a semi-permeable membrane increased after it passed through a magnetic device. The degree of increase observed depended on water flow velocity and was higher at lower flow rates. The increase in permeability observed could be related to a change in the water's surface tension, diffusion velocity, degassing and a more fundamental change in its hydrate clusters' chemical states. These results provide a clear indication that water has been impacted by MWT and demonstrate the degree that water has been impacted by MWT under various flow rates.

Considering the functional similarities between plant cellwall membranes and semi-permeable membranes, as used in this experiment, it is reasonable to conclude that the historical benefits seen with MWT in field studies and in practical agricultural applications may be related to its ability to enhance the transport of water and nutrients into and through plant cells.

This research did not receive any specific grant from funding agencies in the public, commercial, or not-for-profit sectors.

\section{References}

[1] Bogatin J. 1999. Magnetic Treatment of irrigation Water: Experimental Results and application Conditions. Environmental Science Technologies 33: 1280-1285.

[2] Chang K and Weng C. 2006. The effect of an external magnetic field on the structure of liquid water using molecular dynamics simulation. Journal of Applied Physics 100, 043917.

[3] Cho Y I., Lee S. 2005. Reduction of the surface tension of water due to physical water treatment for fouling control in heat exchangers. International Communication in Heat and Mass Transfer 32: 1-9.

[4] Amiri MC, Dadkhah AA. 2006. On the reduction in the surface tension of water due to magnetic treatment. Colloids and Surfaces A: Physiochem. Eng. Aspects 278: 252-255.

[5] Gang N, St-Pierre LS, Persinger MA. 2012. Water dynamics following treatment by one hour 0.16 Tesla static magnetic fields depend on exposure volume. Water 3: 122-131.

[6] Joshi KM, Kamat PV. 1966. Effect of magnetic field on the physical properties of water. J. Ind. Chem. Soc. 43: 620-622.

[7] Holysz L, Chibowski M, Chibowski E. 2002. Time dependent changes of zeta potential and other parameters of in situ calcium carbonate due to magnetic field treatment. Colloids Surf. A 208: 231-240.

[8] Otsuka I, Ozeki S. 2006. Does Magnetic Treatment of water Change Its Properties? The Journal of Physical Chemistry B Letters 110: 1509-1512. 
[9] Majeed AD, Salman SM. 2006. A study of the effect of magnetic field on the absorption spectrum of distilled water. Al-Fateh Journal 27: 8 pp.

[10] Hosoda H, Mori H, Sogoshi N, Nagasawa A, Nakabayashi S. 2003. Refractive indeces of water and aqueous electrolyte solutions under high magnetic fields. The Journal of Physical Chemistry 108: 1461-1464.

[11] Kronenberg KJ. 1985. Magnetic water treatment demystified. Magnets Magazine 6-27.

[12] Noran R, Shani U, Lin I. 1996. The effect of irrigation with magnetically treated water on the translocation of minerals in the soil. Magnetic and Electrical Separation 7: 109-122.

[13] Surendran U, Sandeep O, Joseph EJ. 2016. The impact of magnetic treatment of irrigation water on plant, water and soil characteristics. Agricultural Water Management 178: 21-29.

[14] Hizayn M, Qados AMSA. 2010. Irrigation with magnetized water enhances growth, chemical constiturnt and yield of chickpea. Agriculture and Biology Journal of North America 1: 671-676.

[15] Yazied AE, El-Gizawy A, Khalf SM, El-Satar A, Shalaby OA. 2012. Effect of magnetic field treatments for seeds and irrigation water as well as $\mathrm{N}, \mathrm{P}$ and $\mathrm{K}$ levels on productivity of tomato plants. Journal of Applied Science Research 8 (4): 2088-2099.
[16] Aoda MA, Fattah MA. 2011. The interaction of water magnetic treatment and deficit irrigation on plants productivity and water use efficiency of corn (Zea Mays L.). The Iraqi Journal of Agricultural Science 42: 164-179.

[17] Yadollahpour A, Samaneh R, Kavakebian F. 2014. Application of magnetic water technology in farming and agriculture development: a review of recent advances. Current world Environment 9(3): 695-703.

[18] Ahmed MEM, Abd El-Kader NI. 2016. The influence of magnetic water regimes on soil salinity, growth, yield and tubers quality of potato plants. Middle East Journal of Agriculture Research 5(2): 132-143.

[19] Haq Z, Iqbal M, Jamil Y, Anwar H, Younis A, Arif M, Fareed MZ, Hussain F. 2016. Magnetically treated water irrigation effect on turnip seed germination, seedling growth and enzymatic activities. Information processing in agriculture 3: 99-106.

[20] Benejad H., Abdosalehi E. 2009. The effect of magnetic fields on water hardness reducing. Thirteen International Technology Conference, IWTC 13: 117-128.

[21] Kobe S, Drazic G, Cefalas AC, Sarantopoulou E, Strazisar J. 2002. Nucleation and crystallization of $\mathrm{CaCO}_{3}$ in applied magnetic fields. Crystal Engineering 5: 243-253.

[22] Bondarenko N, Gak EZ. 1995. Electromagnetic hydrophisics and phenomena in the nature. V.1, St. Petersburg's Agrarian University Press, St. Petersburg. 